Quim. Nova, Vol. 35, No. 8, 1510-1516, 2012

\title{
ALKALINE EARTH LAYERED BENZOATES AS REUSABLE HETEROGENEOUS CATALYSTS FOR THE METHYL ESTERIFICATION OF BENZOIC ACID
}

\author{
Swamy Arêa Maruyama, Fabio da Silva Lisboa, Luiz Pereira Ramos e Fernando Wypych* \\ Departamento de Química, Universidade Federal do Paraná, CP 19081, Centro Politécnico, 81531-980 Curitiba, PR - Brasil
}

Recebido em 9/8/11; aceito em 9/4/12; publicado na web em $2 / 7 / 12$

\begin{abstract}
This paper describes the synthesis and characterization of layered barium, calcium and strontium benzoates and evaluates the potential of these materials as catalysts in the synthesis of methyl benzoate. The methyl esterification of benzoic acid was investigated, where the effects of temperature, alcohol:acid molar ratio and amount of catalyst were evaluated. Ester conversions of 65 to $70 \%$ were achieved for all the catalysts under the best reaction conditions. The possibility of recycling these metallic benzoates was also demonstrated, evidenced by unaltered catalytic activity for three consecutive reaction cycles.
\end{abstract}

Keywords: layered benzoates; esterification; benzoic acid.

\section{INTRODUCTION}

Methyl benzoate, an ester derived from benzoic acid, is an important intermediate for the production of dyes, flavoring compounds, plasticizers, perfumes, pesticides and mold growth biomarkers. It is also used to produce higher esters by transesterification, in which methyl benzoate reacts with higher boiling point alcohols, such as butanol and benzyl alcohol, to produce benzyl or butyl benzoate and methanol as a secondary product. Butyl benzoate is particularly used as a plasticizer, while benzyl benzoate is an excellent perfume ingredient, candy flavoring compound and insect repellent. ${ }^{1-9}$

Methyl benzoate can be synthesized in many ways, but Fischer's esterification is the simplest and most widely used industrial method. ${ }^{9}$ This method consists of adding concentrated sulfuric acid as a homogeneous catalyst to a mixture of benzoic acid and methanol in excess. ${ }^{7}$ As a result, high ester conversions are achieved in relatively short times compared to the time required for this same reaction using heterogeneous acid catalysts. However, there are some major drawbacks of sulfuric acid catalysis, including the generation of other oxidation and hydrolysis byproducts, equipment corrosion, the need for extensive purification procedures and the production of large volumes of acid waste, causing environmental problems. ${ }^{8-10}$

To meet the increasing economic and ecological challenges of green chemistry, it is important to develop an alternative process that combines high productivity with reduced waste generation, replacing conventional acid catalysts with eco-friendly materials. In this sense, the development of heterogeneous catalysts can contribute significantly to this process, since they can be easily recovered and reused, yielding purer products in a more environmentally benign industrial process. Layered carboxylates have been successfully used as heterogeneous catalysts for the esterification of fatty acids and transesterification of vegetable oils. ${ }^{11}$ However, to the best of our knowledge, studies involving layered benzoates as catalysts for the methyl esterification of benzoic acid have not yet been reported in the specialized literature.

In this work, aryl-carboxylate derivatives of benzoic acid with the metals barium, calcium and strontium were synthesized and their catalytic activity evaluated in the methyl esterification of benzoic acid.

*e-mail: wypych@ufpr.br
Studies were also carried out to evaluate the potential reuse of these catalysts in at least three consecutive reaction cycles.

\section{EXPERIMENTAL}

\section{Synthesis of layered benzoates}

Layered benzoates were synthesized by chemical precipitation using analytical grade reagents. ${ }^{12} \mathrm{~A}$ volume of 66.2 mmoles of benzoic acid was completely dissolved in $40 \mathrm{~mL}$ of methanol and neutralized under stoichiometric conditions with 66.2 mmoles of sodium hydroxide, which was previously dissolved in methanol by heating at 50 ${ }^{\circ} \mathrm{C}$ under vigorous stirring. The white precipitate of sodium benzoate was dissolved by adding $50 \mathrm{~mL}$ of distilled water at room temperature under magnetic stirring.

Barium benzoate was obtained as a white solid precipitate by slowly adding a dilute solution of barium chloride dihydrate (Vetec, $98.0 \%$ ) to the sodium benzoate solution under magnetic stirring. The former solution was prepared by adding 33.1 mmoles of barium chloride dihydrate to $100 \mathrm{~mL}$ of distilled water. In the syntheses of calcium and strontium benzoates, the barium chloride solution was replaced by a solution containing 33.1 mmoles of either anhydrous calcium chloride (Vetec, $98.0 \%$ ) or strontium chloride dihydrate (Merck, 98.0\%) in $100 \mathrm{~mL}$ of distilled water.

The freshly synthesized white solids were centrifuged, washed extensively with distilled water and dried in a vacuum oven at a temperature of $60{ }^{\circ} \mathrm{C}$ until constant weight. Finely divided white powders were obtained and identified as $\mathrm{BaBZ}, \mathrm{CaBZ}$ and $\mathrm{SrBZ}$ for the corresponding $\mathrm{Ba}, \mathrm{Ca}$ and $\mathrm{Sr}$ benzoates, respectively.

\section{Esterification reactions}

The catalytic activity of metal benzoates was evaluated in the esterification of benzoic acid P.A. (98\%) using methanol P.A. (99.8\%). The esterification reactions were performed in a Büchiglass Miniclave Drive pressurized steel reactor, based on the following variables: methanol:benzoic acid molar ratio (MR), the weight percentage of catalyst in relation to the total mass of benzoic acid (CAT), and the reaction temperature $(\mathrm{T})$ (see Table 1 for details). The reaction vessel was sealed but not pressurized so that the internal pressure corresponded to the vapor pressure of the methanol. To check the repeatability 
of the method, specific conditions of the reactions (temperature of $140{ }^{\circ} \mathrm{C}, \mathrm{MR}$ of $10: 1$ and catalysts amount of $6 \%$ ) were evaluated in three replicates. ${ }^{13}$

A systematic study was performed, where the temperature was varied from 120 to $160{ }^{\circ} \mathrm{C}$ (in steps of $20^{\circ} \mathrm{C}$ ), MR from 6:1, 10:1 and $14: 1$ and catalyst amount (CAT) from 2, 6 and $10 \%$. Conversion of benzoic acid to methyl benzoate was measured by changes in acid number using the Ca-5a-40 method of the American Oil Chemists' Society. ${ }^{14}$

Recycling experiments were carried out under the conditions in which the highest ester conversions were obtained. Also, the solid catalyst was characterized after each reaction cycle to evaluate the integrity of its layered structure. For this purpose, part of the solid catalyst, recovered after reaction completion, was separated, washed with acetone and distilled water then dried under vacuum at $60{ }^{\circ} \mathrm{C}$ until constant weight. A second reaction cycle was carried out with the remaining solid catalyst under identical conditions of temperature, time and MR as well as total reaction volume. This entire procedure was repeated once again for a total of three recycling stages, from which three aliquots of the spent catalyst were reserved for analysis.

Thermal conversions were also carried out in this study to serve as reaction controls, in which time and temperature were investigated under the same conditions as described above. Three replicates were also carried under intermediary reaction conditions to verify the reliability of the experimental results. ${ }^{13}$

\section{Characterization of solid catalysts}

The X-Ray powder diffraction (XRPD) experiments were conducted in a Shimadzu XDR-6000 device with the Bragg Brentano geometry, using $\mathrm{CuK}_{\alpha}$ radiation of $\lambda=1.5418 \AA$, current of $30 \mathrm{~mA}$, tension of $40 \mathrm{kV}$ and a dwell time of $2^{\circ} \mathrm{min}^{-1}$. The samples were placed in glass sample holders and lightly hand pressed so that the crystals were perfectly set in the holder's plane.

FTIR measurements were made in $\mathrm{KBr}$ (spectroscopic grade, Vetec) discs and collected in a Bio-Rad FTS 3500GX spectrophotometer, in the 400 to $4000 \mathrm{~cm}^{-1}$ range, with a resolution of $4 \mathrm{~cm}^{-1}$ and accumulation of 32 scans.

The thermal analysis measurements were performed on a MettlerToledo TG/SDTA 851 device under oxygen flow of $50 \mathrm{~mL} \mathrm{~min}{ }^{-1}$, using platinum crucibles, heating rate of $10^{\circ} \mathrm{C} \mathrm{min}^{-1}$ and temperatures ranging from 30 to $1000^{\circ} \mathrm{C}$.

\section{RESULTS AND DISCUSSION}

\section{Characterizations of layered benzoates}

A comparison between the X-Ray diffraction patterns of benzoic acid and layered benzoates (Figure 1) demonstrated that the synthesized solids were not contaminated by benzoic acid or any of the starting chlorides used in their synthesis.

Both barium and strontium benzoates showed similarities in their layered structural organization. Therefore, these compounds are probably isostructural with slight differences in basal plane distances, due to different ionic radii. Calcium benzoate is different from the others in that it does not present a uniform sequence of basal diffraction peaks, due to the different crystal morphologies. ${ }^{15-18}$ To calculate the basal distances of the synthesized benzoates, the peak of the highest possible order was used and the values calculated for BaBZ, SrBZ and $\mathrm{CaBZ}$ were $15.07,15.73$ and $16.5 \AA$, respectively. These values are consistent with the existence of double benzoate anion layers between metal centers, along the crystallographic axis "b" ${ }^{15}$ taking the structure of calcium benzoate trihydrate as a reference (Figure 2). ${ }^{16,17}$
In calcium benzoate trihydrate, the structuring of the layer occurs by the tetrahedral coordination of benzoate groups and the metal

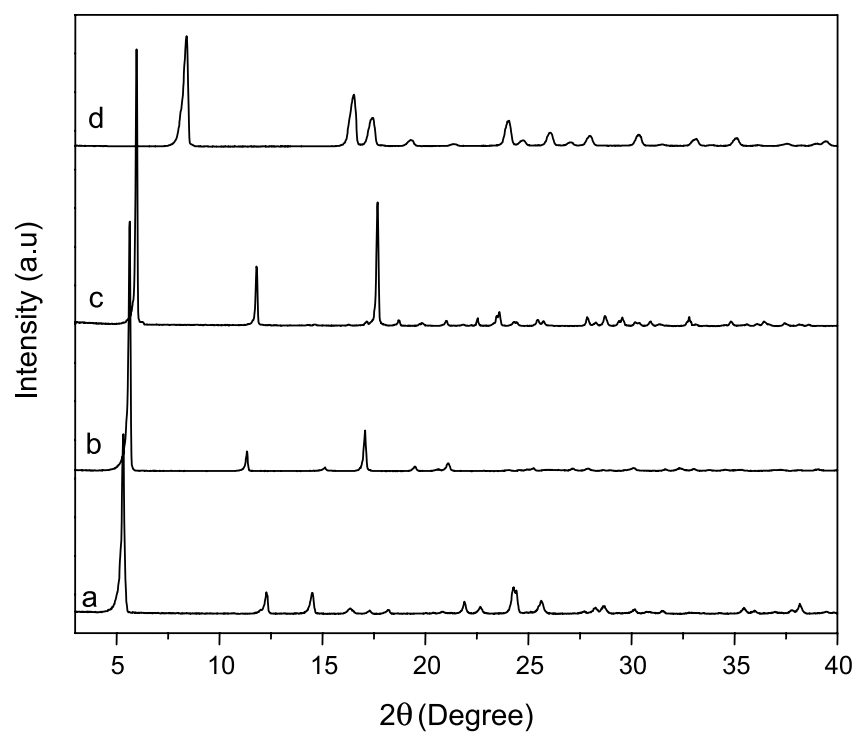

Figure 1. X-ray powder diffraction patterns of the synthesized layered benzoates, $\mathrm{CaBz}(a), \mathrm{SrBz}(b)$ and $\mathrm{BaBz}(\mathrm{c})$, and benzoic acid (d)

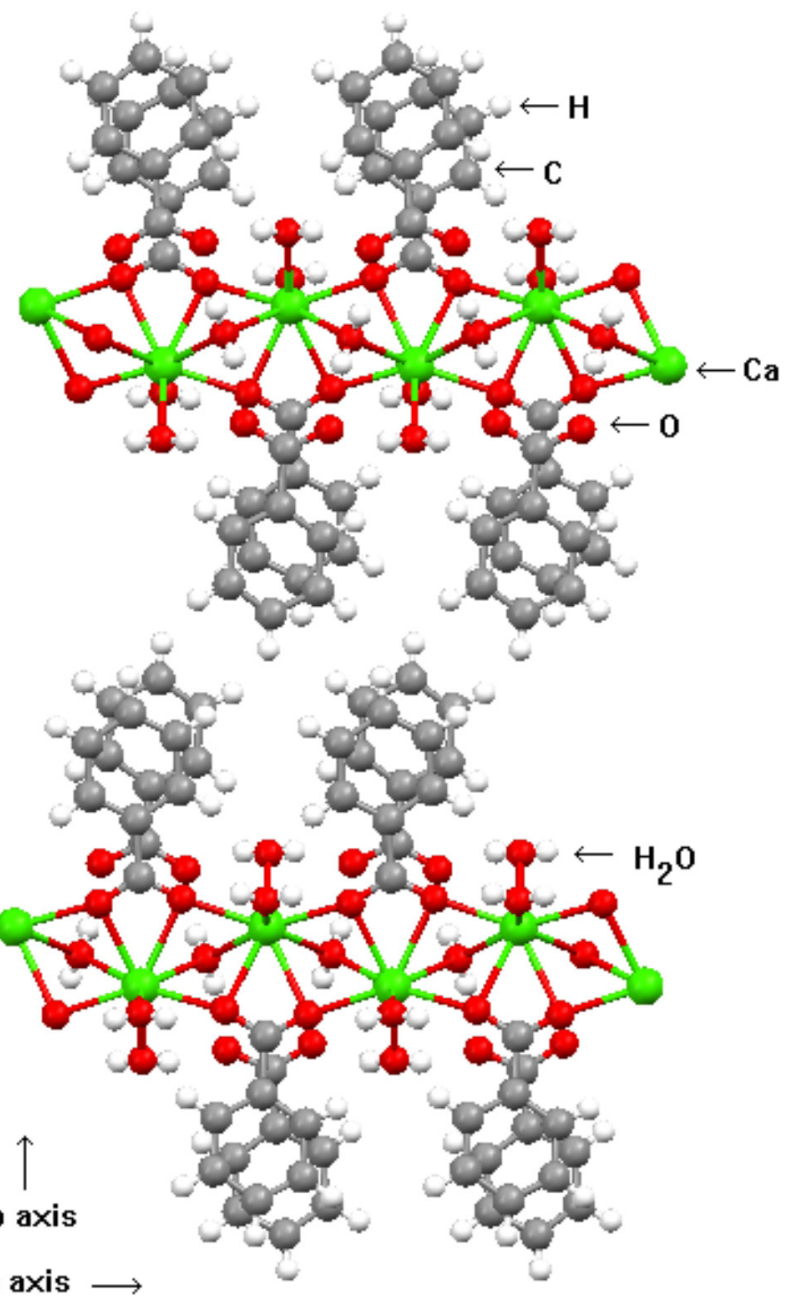

Figure 2. Layer structure of calcium benzoate trihydrate (vision in the $b x c$ plane of the structure) 
centers (Figure 2). This coordination can be easily verified by the FTIR spectra of the compounds, where there is a clear predominance of the vibrational modes that are characteristic of the aromatic rings of benzoic acid, along with the asymmetrical and symmetrical stretching of $\mathrm{C}=\mathrm{O}$ of carboxylate anions, which are coordinated to the metal centers (Figure 3).

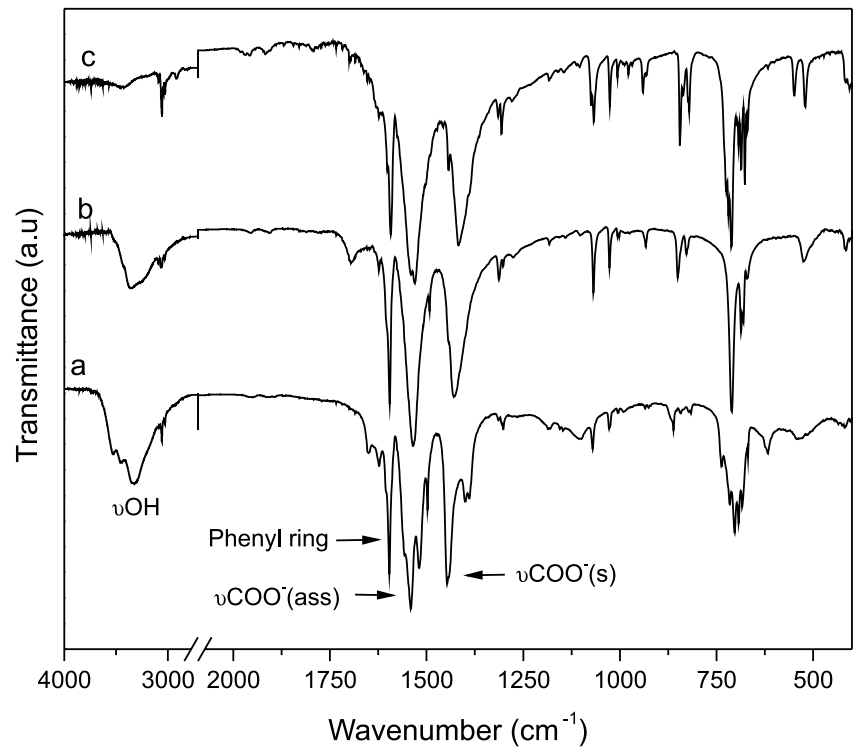

Figure 3. FTIR spectra of the synthesized benzoates. $\operatorname{SrBZ}(a) ; \mathrm{CaBZ}(b)$ and $\mathrm{BaBZ}(\mathrm{c})$

The FTIR spectra of barium, calcium and strontium benzoates displayed the stretching vibrational modes of C-H bonds at 3059, 3060 and $3066 \mathrm{~cm}^{-1}$, respectively, aromatic C-C vibration modes at $1590 \mathrm{~cm}^{-1}$ (all at the same wavenumber) and very sharp absorption bands at 713, 700 and $713 \mathrm{~cm}^{-1}$, due to the out-of-plane $\mathrm{C}-\mathrm{H}$ vibration. ${ }^{15}$ The $\mathrm{C}=\mathrm{O}$ bond vibration from the carboxylate group are those that most contribute to understanding the structure formation in layered metal benzoates. ${ }^{18-20}$ The asymmetrical stretching values at 1517,1540 and $1539 \mathrm{~cm}^{-1}$, and symmetrical stretching values of the carboxylate group at 1420, 1446 and $1429 \mathrm{~cm}^{-1}$ for barium, calcium and strontium benzoates, respectively, provide information about the coordination mode of the carboxylate to the metal center. The difference in wavenumber between the symmetrical and asymmetrical stretching $(\Delta v)$, with values of 97,94 and $110 \mathrm{~cm}^{-1}$ for barium, calcium and strontium benzoates, respectively, indicates that the carboxylate group is coordinated in bridge form between two metal centers. ${ }^{15,19-21}$ Therefore, the layer structure is formed by the interaction between the oxygen present in the extremity of the carboxylate anion with a different metal center of barium, calcium or strontium, as observed in Figure 2, which depicts the proposed similar structures of the synthesized compounds.

The thermogravimetric (TGA) and differential thermal (DTA) analyses of the synthesized layered materials were shown to be similar to that originally observed for barium benzoate (Figure 4). ${ }^{18}$

In the DTG curve of BaBZ (Figure 4), the small endothermic peak in the region of $385^{\circ} \mathrm{C}$ was attributed to the melting of the solid material. Between 400 and $555^{\circ} \mathrm{C}$, a mass loss of $47.4 \%$ observed in the TGA curve, accompanied by an exothermic peak at $540{ }^{\circ} \mathrm{C}$ in the DTA curve, represents the oxidation of the majority of the organic part of the benzoate structure. Finally, the beginning of decomposition of barium carbonate was only observed above $880^{\circ} \mathrm{C}$ and was incomplete at $1000{ }^{\circ} \mathrm{C}$. Based on these observations, the composition of anhydrous barium benzoate was stipulated as $\mathrm{Ba}\left[\mathrm{C}_{6} \mathrm{H}_{5} \mathrm{COO}\right]_{2}$, which

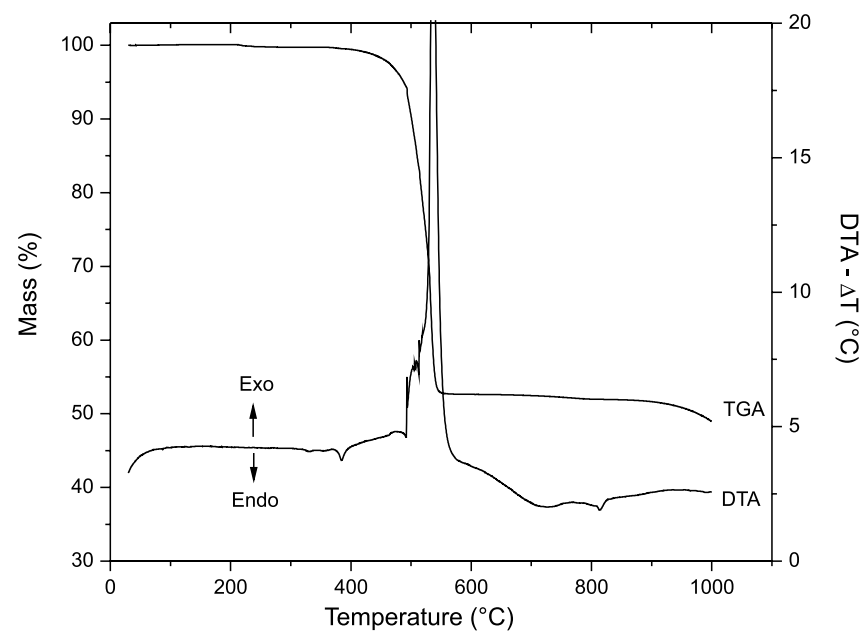

Figure 4. Thermal analysis curves of the synthesized barium benzoates - BaBZ

is in agreement with research data obtained elsewhere. ${ }^{18}$

In the TGA curve of CaBZ (Figure $1 \mathrm{~S}$, supplementary material), a dehydration mass loss of $15.3 \%$ was observed at temperatures up to $101{ }^{\circ} \mathrm{C}$ and this event was associated with an endothermic peak in the corresponding DTA curve. This moisture content was also consistent with the occurrence of $\mathrm{O}-\mathrm{H}$ absorption bands in the FTIR spectrum of $\mathrm{CaBZ}$, which are usually attributed to hydrogen bonding involving water molecules. Oxidation of the organic part of the benzoate structure was observed between 450 and $540{ }^{\circ} \mathrm{C}$ with an additional mass loss of $52.6 \%$ in the TGA curve. This was accompanied by an exothermic peak at $534{ }^{\circ} \mathrm{C}$ in the DTA curve. The decomposition of $\mathrm{CaCO}_{3}$ began at $547^{\circ} \mathrm{C}$ and was complete only at temperatures above $800{ }^{\circ} \mathrm{C}$, as can be seen in the TGA curve. With the results obtained by thermal analysis, the composition of trihydrated calcium benzoate was defined as $\mathrm{Ca}\left[\mathrm{C}_{6} \mathrm{H}_{5} \mathrm{COO}\right]_{2} \cdot 3 \mathrm{H}_{2} \mathrm{O}$, which again is in agreement with the data available in the literature. ${ }^{22}$

Finally, the TGA profile of SrBZ (Figure 2S, supplementary material) revealed the occurrence of a mass loss of $4.4 \%$ up to 190 ${ }^{\circ} \mathrm{C}$ in the TGA curve, which was associated with an endothermic peak at $170{ }^{\circ} \mathrm{C}$ in the DTA curve. This event was attributed once again to the loss of water present in the composition of metal carboxylate. Between 437 and $559^{\circ} \mathrm{C}$, another event led to a mass loss of $45.7 \%$ in the TGA curve, where this event was accompanied by an exothermic peak at $535{ }^{\circ} \mathrm{C}$ in the DTA curve, whose cause was the oxidation of the majority of the organic part of the strontium benzoate structure, forming strontium carbonate. The decomposition of strontium carbonate to $\mathrm{SrO}$ began at $770{ }^{\circ} \mathrm{C}$ with a further mass loss of $22.6 \%$ in the TGA curve, without association with any visible thermal processes in the DTA curve. Based on these observations, the composition of strontium benzoate was stipulated as $\mathrm{Sr}\left[\mathrm{C}_{6} \mathrm{H}_{5} \mathrm{COO}\right]_{2} \cdot \mathrm{H}_{2} \mathrm{O}$, which contains a structural water molecule in its composition.

\section{Synthesis of methyl benzoate from benzoic acid}

The use of metal benzoates in the esterification of benzoic acid with methanol was initially tested under reflux at $65^{\circ} \mathrm{C}$. In general, the solid materials were completely solubilized during the process but their recovery could be obtained semi quantitatively after removal of the methanol used in excess and the addition of $5 \mathrm{~mL}$ of acetone. The solubilization of the catalysts was also observed when the reaction was carried out in a closed stainless steel vessel, in which the internal pressure was determined by the most volatile component of the reaction system (methanol). It is important to mention that alcohol acts both as a reagent and solvent of the esterification reagents. The 
process of solvation of reactants and catalyst by methanol probably occurred through the action of various intermolecular forces, such as ion-dipole attraction, Van der Waals forces and hydrogen bonding, while the solubilized benzoate molecules or their solvated clusters were responsible for the observed catalytic activity. ${ }^{23}$ Also, the ease with which the catalysts were recovered varied in the following order: barium, strontium and calcium.

To evaluate the time required to reach reaction equilibrium, the synthesis of methyl benzoate was performed as a function of time in the presence (catalytic conversion) and absence (thermal conversion) of a catalyst (Table 1). The catalyst used was barium benzoate, because this compound was more easily recovered after the end of the reactions, in comparison to the other evaluated benzoates.

Table 1. Results obtained in the production of methyl benzoate catalyzed by barium benzoate in function of time

\begin{tabular}{ccc}
\hline Time (hours) & $\begin{array}{c}\text { Ester }(\%)-\text { Thermal } \\
\text { conversion }\end{array}$ & $\begin{array}{c}\text { Ester }(\%) \text { - Catalyzed } \\
\text { conversion }\end{array}$ \\
\hline 0 & 0 & 0 \\
1 & 33.5 & 40.8 \\
2 & 46.7 & 68.5 \\
3 & 57.0 & 70.5 \\
4 & 69.04 & 73.9 \\
4.5 & 69.06 & 75.2 \\
5 & 69.09 & 75.9 \\
\hline
\end{tabular}

Experimental conditions: Temperature of $160^{\circ} \mathrm{C}$, molar ratio (alcohol:benzoic acid) of $14: 1$ and $10 \%$ of catalyst (in relation to benzoic acid mass).

The zero time indicated in Table 1 corresponds to the instant at which the reagents were introduced at room temperature into the reactor vessel. The reactor temperature was set to reach $160^{\circ} \mathrm{C}$, which took $60 \mathrm{~min}$ to achieve. At this point, the levels of methyl benzoate in the reaction medium were 40.8 and $33.5 \%$ for the processes carried out in the presence and the absence of barium benzoate, respectively. After 270 min of reaction, the conversions obtained in both cases were not statistically different from each other, demonstrating that at this point the system had already reached its chemical equilibrium (Table 1). Since a time of $4.5 \mathrm{~h}$ would be too long whereas a reaction time of $2 \mathrm{~h}$ would already have achieved a higher conversion, the latter time was chosen to evaluate the performance of the catalysts.

The conversion of benzoic acid to methyl benzoate could also be obtained in the absence of a catalyst. This has been referred to as thermal conversion. ${ }^{24}$ Therefore, we carried out tests to quantify this thermal conversion and, by doing so, aimed to evaluate the actual catalytic performance of each metal benzoate or how much of the experimental conversion could be attributed to the catalytic effect of these layered compounds. The difference between the conversion obtained in the presence of catalyst and thermal conversion is treated in this work as a conversion gain (CG) and these values were measured and/or expressed in percentage points (p.p.).

Table 2 shows the percentage of thermal conversion of benzoic acid to methyl benzoate. The highest thermal conversion was $46.70 \%$ at $160{ }^{\circ} \mathrm{C}$, with a molar ratio of $14: 1$, while the lowest was $16.09 \%$ at $120^{\circ} \mathrm{C}$, with a molar ratio of $6: 1$. The results of the center point were very similar, being close to 27 , with a standard deviation of $\pm 0.18 \%$.

The results for the reactions in which the catalyst used was barium benzoate (CG data in Table 3), with the exception of experiments 8 and 11 , were all positive. Therefore, the catalytic activity of barium benzoate as an effective contribution of this compound was characterized in the conversion of benzoic acid into the corresponding methyl ester.
Table 2. Methyl esterification regarding thermal conversion

\begin{tabular}{cccccc}
\hline & Variables & & \multicolumn{2}{c}{ Reaction condition } & Results \\
$\mathrm{E}$ & MR & $\mathrm{T}$ & $\mathrm{MR}$ & $\mathrm{T}\left({ }^{\circ} \mathrm{C}\right)$ & Ester $(\%)$ \\
\hline 1 & -1 & -1 & $6: 1$ & 120 & 16.09 \\
2 & -1 & +1 & $6: 1$ & 160 & 39.01 \\
3 & +1 & -1 & $14: 1$ & 120 & 23.62 \\
4 & +1 & +1 & $14: 1$ & 160 & 46.70 \\
5 & 0 & 0 & $10: 1$ & 140 & 27.77 \\
6 & 0 & 0 & $10: 1$ & 140 & 27.56 \\
7 & 0 & 0 & $10: 1$ & 140 & 27.40 \\
\hline
\end{tabular}

Exp = experiment; MR = Molar Ratio (alcohol:benzoic acid); $\mathrm{T}=\mathrm{Tem}$ perature; reaction time was established at $2 \mathrm{~h}$; standard deviation of \pm 0.18 .

Table 3. Methyl esterification using BaBZ as catalyst

\begin{tabular}{ccccccccc}
\hline & \multicolumn{3}{c}{ Variables } & \multicolumn{3}{c}{ Reaction condition } & \multicolumn{2}{c}{ Results } \\
Exp & MR & CAT & T & MR & $\begin{array}{c}\text { CAT } \\
(\%)\end{array}$ & $\begin{array}{c}\text { T } \\
\left({ }^{\circ} \mathrm{C}\right)\end{array}$ & $\begin{array}{c}\text { Ester } \\
(\%)\end{array}$ & $\begin{array}{c}\text { CG } \\
\text { (Ester p.p. })\end{array}$ \\
\hline 8 & -1 & -1 & -1 & $6: 1$ & 2 & 120 & 13.59 & -2.50 \\
9 & -1 & -1 & +1 & $6: 1$ & 2 & 160 & 50.13 & 11.12 \\
10 & -1 & +1 & -1 & $6: 1$ & 10 & 120 & 29.56 & 13.47 \\
11 & +1 & -1 & -1 & $14: 1$ & 2 & 120 & 23.39 & -2.23 \\
12 & +1 & -1 & +1 & $14: 1$ & 2 & 160 & 56.73 & 10.03 \\
13 & +1 & +1 & -1 & $14: 1$ & 10 & 120 & 31.67 & 8.05 \\
14 & -1 & +1 & +1 & $6: 1$ & 10 & 160 & 65.49 & 26.48 \\
15 & +1 & +1 & +1 & $14: 1$ & 10 & 160 & 68.52 & 21.82 \\
16 & 0 & 0 & 0 & $10: 1$ & 6 & 140 & 37.23 & 9.46 \\
17 & 0 & 0 & 0 & $10: 1$ & 6 & 140 & 37.44 & 9.88 \\
18 & 0 & 0 & 0 & $10: 1$ & 6 & 140 & 37.51 & 10.11 \\
\hline
\end{tabular}

Exp $=$ experiment $; \mathrm{MR}=$ molar ratio $; \mathrm{CAT}=$ catalyst $\mathrm{T}=$ temperature $; \mathrm{CG}$ $=$ Conversion obtained in the presence of catalyst - thermal conversion; time of $2 \mathrm{~h}$; standard deviation of \pm 0.22 ; pp = percentage point.

The best conversions were obtained at $160{ }^{\circ} \mathrm{C}$ with a catalyst loading of $10 \%$ in relation to the mass of benzoic acid. Under these conditions, 68.52 and $65.49 \%$ of the benzoic acid was converted to methyl benzoate using methanol:benzoic acid molar ratios of 14:1 and 6:1, respectively, indicating conversion gains (CG) of 26.48 and 21.82 p.p. in relation to the thermal conversion alone. On the other hand, by expressing CG in relation to the amount of added catalyst, based on experiments in which MR and T were kept constant (experiments 12 and 15), better performance was observed at lower catalyst loadings $(10.03 / 2=5.00 v s .21 .82 / 10=2.18)$. This trend was also observed for the other two catalysts, suggesting structural similarities of the evaluated catalysts.

The results obtained for the methyl esterification of benzoic acid employing calcium benzoate as the catalyst are shown in Table 4.

The catalyst performance of $\mathrm{CaBZ}$ led to positive $\mathrm{CT}$ values under all experimental conditions tested in this study. The best conversions, of 66.84 and $64.26 \%$, were obtained in experiments 25 and 26, respectively, where molar ratios of $6: 1$ and 14:1 were applied at $160{ }^{\circ} \mathrm{C}$ with a catalyst loading of $10 \%$. Also, by comparing the conversion derived from experiments 23 and 12 , CaBZ was shown to have better catalytic performance at lower catalyst loadings and higher MR, because the CG value of the former was greater than that of the latter when expressed in relation to the amount of catalyst 
Table 4. Methyl esterification using $\mathrm{CaBZ}$ as catalyst

\begin{tabular}{ccccccccc}
\hline & \multicolumn{3}{c}{ Variables } & \multicolumn{3}{c}{ Reaction conditions } & \multicolumn{2}{c}{ Results } \\
Exp & MR & CAT & T & MR & $\begin{array}{c}\text { CAT } \\
(\%)\end{array}$ & $\begin{array}{c}\mathrm{T} \\
\left({ }^{\circ} \mathrm{C}\right)\end{array}$ & $\begin{array}{c}\text { Ester } \\
(\%)\end{array}$ & (Ester p.p.) \\
\hline 19 & -1 & -1 & -1 & $6: 1$ & 2 & 120 & 21.32 & 5.23 \\
20 & -1 & -1 & +1 & $6: 1$ & 2 & 160 & 57.93 & 18.92 \\
21 & -1 & +1 & -1 & $6: 1$ & 10 & 120 & 31.25 & 15.16 \\
22 & +1 & -1 & -1 & $14: 1$ & 2 & 120 & 25.61 & 1.99 \\
23 & +1 & -1 & +1 & $14: 1$ & 2 & 160 & 61.64 & 14.94 \\
24 & +1 & +1 & -1 & $14: 1$ & 10 & 120 & 35.52 & 11.90 \\
25 & -1 & +1 & +1 & $6: 1$ & 10 & 160 & 64.26 & 25.25 \\
26 & +1 & +1 & +1 & $14: 1$ & 10 & 160 & 66.84 & 20.14 \\
27 & 0 & 0 & 0 & $10: 1$ & 6 & 140 & 44.99 & 17.42 \\
28 & 0 & 0 & 0 & $10: 1$ & 6 & 140 & 45.09 & 17.52 \\
29 & 0 & 0 & 0 & $10: 1$ & 6 & 140 & 45.78 & 18.21 \\
\hline
\end{tabular}

Exp $=$ experiment $; \mathrm{MR}=$ Molar Ratio $; \mathrm{CAT}=$ Catalyst $; \mathrm{T}=$ temperature $; \mathrm{CG}$ $=$ Conversion obtained in the presence of catalyst - thermal conversion; time of $2 \mathrm{~h}$; standard deviation of \pm 0.43 .

used for conversion $(14.94 / 2=7.47$ vs. $10.03 / 2=5.00$, respectively)

Anhydrous calcium benzoate was also tested under the same conditions used in experiments 25 and 26 (Table 4) and conversions of 74.42 and $76.13 \%$ were obtained, which are all higher than those observed for the same tests with calcium benzoate trihydrate, demonstrating the greater number of active sites when the same mass of catalyst was used.

The last carboxylate tested in this study was strontium benzoate (Table 5). Like BaBZ and CaBZ, the catalytic activity of this compound was highest when esterification was carried out at $160{ }^{\circ} \mathrm{C}$ with $10 \%$ catalyst in relation to the benzoic acid mass. Benzoic acid conversions of 67.91 and $65.32 \%$ were obtained when methanol:benzoic acid molar ratios of 14:1 to $6: 1$ were used, respectively, which translated into CG gains of 21.21 and 26.31 p.p. when compared to the control. Once again, when CG was expressed in relation to the amount of catalyst used for conversion, the best performance was observed at the lowest catalyst loading $(13.42 / 2=6.50$ for experiment $45 \mathrm{vs}$. $21.21 / 10=2.12$ for experiment 48$)$.

Table 5. Methyl esterification using SrBZ as catalyst

\begin{tabular}{ccccccccc}
\hline \multicolumn{3}{c}{ Variables } & \multicolumn{3}{c}{ Reaction conditions } & \multicolumn{2}{c}{ Results } \\
Exp & MR & CAT & T & MR & $\begin{array}{c}\text { CAT } \\
(\%)\end{array}$ & $\begin{array}{c}\text { T } \\
\left({ }^{\circ} \mathrm{C}\right)\end{array}$ & $\begin{array}{c}\text { Ester } \\
(\%)\end{array}$ & $\begin{array}{c}\text { CG } \\
\text { (Ester p.p.) }\end{array}$ \\
\hline 41 & -1 & -1 & -1 & $6: 1$ & 2 & 120 & 27.89 & 11.80 \\
42 & -1 & -1 & +1 & $6: 1$ & 2 & 160 & 58.66 & 19.65 \\
43 & -1 & +1 & -1 & $6: 1$ & 10 & 120 & 33.65 & 17.57 \\
44 & +1 & -1 & -1 & $14: 1$ & 2 & 120 & 31.45 & 7.83 \\
45 & +1 & -1 & +1 & $14: 1$ & 2 & 160 & 60.12 & 13.42 \\
46 & +1 & +1 & -1 & $14: 1$ & 10 & 120 & 35.99 & 12.37 \\
47 & -1 & +1 & +1 & $6: 1$ & 10 & 160 & 65.32 & 26.31 \\
48 & +1 & +1 & +1 & $14: 1$ & 10 & 160 & 67.91 & 21.21 \\
49 & 0 & 0 & 0 & $10: 1$ & 6 & 140 & 42.37 & 14.79 \\
50 & 0 & 0 & 0 & $10: 1$ & 6 & 140 & 42.85 & 15.31 \\
51 & 0 & 0 & 0 & $10: 1$ & 6 & 140 & 42.56 & 14.99 \\
\hline
\end{tabular}

Exp $=$ experiment $; \mathrm{MR}=$ Molar Ratio $; \mathrm{CAT}=$ Catalyst $; \mathrm{T}=$ temperature $; \mathrm{CG}$ $=$ Conversion obtained in the presence of catalyst - thermal conversion; time of $2 \mathrm{~h}$; standard deviation of \pm 0.3 .

\section{Characterization of catalysts after methyl esterification of benzoic acid}

To evaluate the chemical integrity of the catalyst after reaction completion, the remaining solids were recovered by filtration, washed extensively with acetone and water, oven-dried at $60{ }^{\circ} \mathrm{C}$ to constant weight and analyzed by XRPD (Figure 5 and Figures $3 \mathrm{~S}$ and $4 \mathrm{~S}$, supplementary material). In all cases, the catalysts retained their layered structure with the same basal spacing of the original compounds, even though slight changes were observed with regard to their structural order.

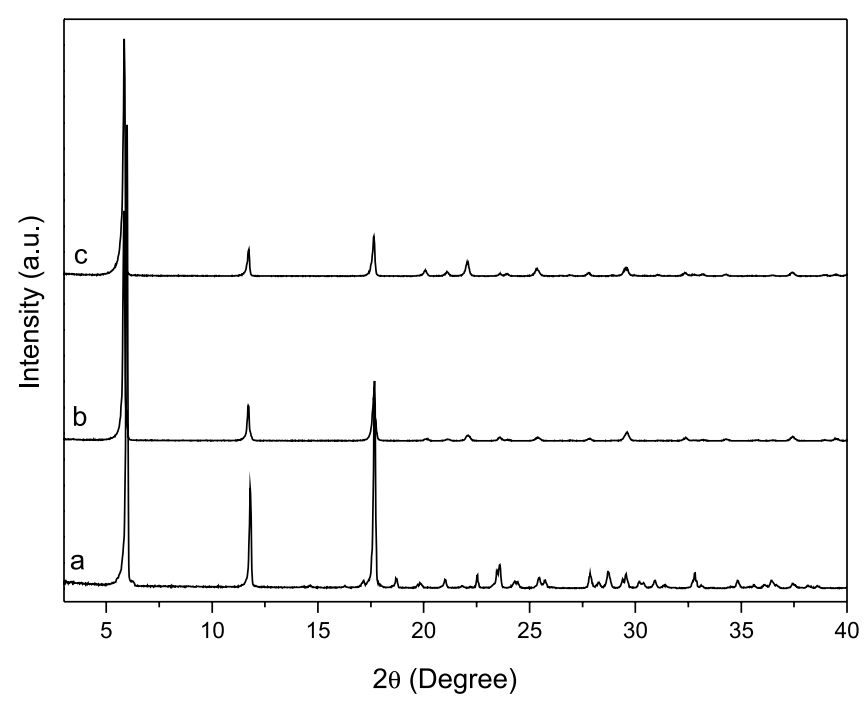

Figure 5. X-ray diffraction patterns of barium benzoate before (a) and after two reaction cycles $(b, c)$

The small decrease in the crystallinity of CaBZ (Figure 3S, supplementary material) and SrBZ (Figure 4S, supplementary material) can be attributed to the restructuring of layers that were not perfectly stacked, ${ }^{25}$ as well as to the different times to which the reused benzoates were subjected in the new crystallization process. On the other hand, the FTIR spectra of the solids recovered after esterification of benzoic acid with methanol (Figure 6 and Figures $5 \mathrm{~S}$ and $6 \mathrm{~S}$, supplementary material) showed no significant change in the vibrational modes first seen in the original compounds, suggesting that the catalysts retained their structural integrity after reaction completion (Figure 3).

\section{Catalyst reuse in the methyl esterification of benzoic acid}

The conversion values obtained after the first and second reuse of $\mathrm{BaBZ}$, under the conditions used in experiment 15 , were 66.78 and $63.77 \%$, respectively (Figure 7). These results showed that BaBZ maintained its catalytic activity for at least three reaction cycles, albeit with a slight decrease in the conversion values obtained. This was probably due to physical losses during catalyst recovery and recycling. Therefore, the total volume of the reaction media had to be slightly decreased in order to keep everything else unchanged, and since the reactor volume remained the same there was a higher amount of methanol in the reactor head space, leading to a proportionally lower amount of methanol in direct contact with benzoic acid and to the observed decrease in benzoic acid conversion at the end of the reaction.

To check the validity of this assumption, tests were carried out in which the total reaction volume was also kept unchanged, along with all other reaction parameters (Figure 7). In these tests, calcium 


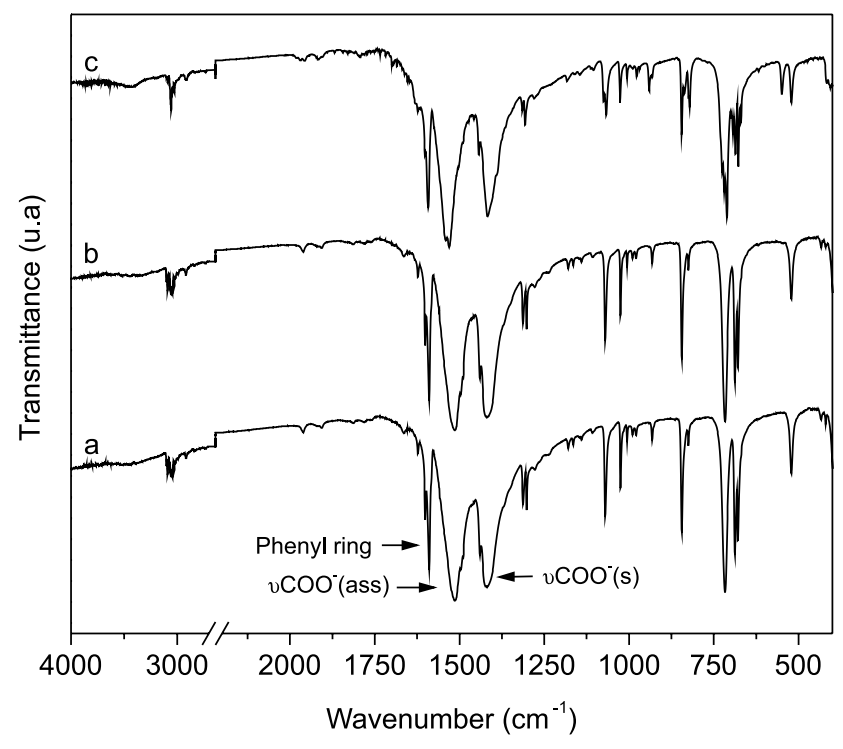

Figure 6. FTIR spectra of barium benzoate before (a) and after two reaction cycles $(b, c)$

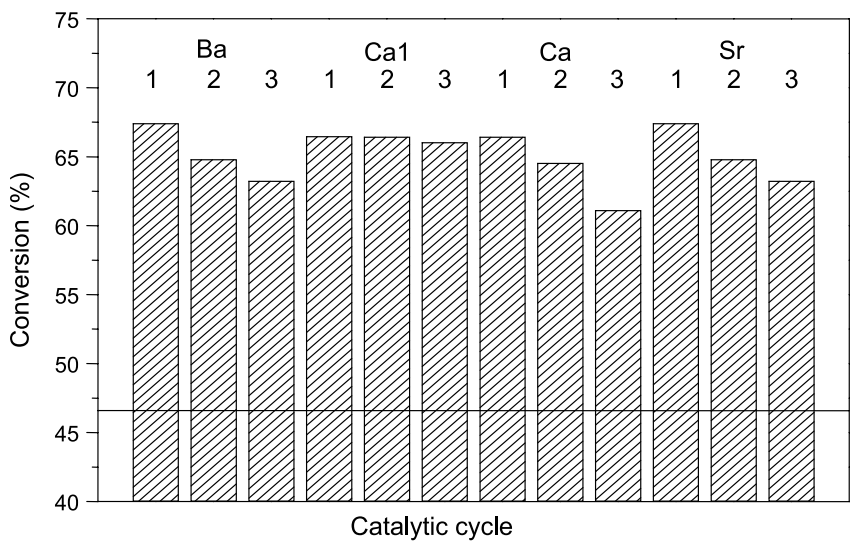

Figure 7. Reuse tests of layered benzoates in three consecutive reaction cycles (1 to 3). Cal- CaBZ when using the same amount of catalyst and reagents of the first use cycle; $\mathrm{BaBZ}, \mathrm{CaBZ}$ and $\mathrm{SrBZ}$ : the reagents were recalculated in relation to the catalysts amount recovered. The solid line crossing the " $y$ " axis is related to the thermal conversion

benzoate was employed as the catalyst because this showed the most pronounced decrease in catalytic activity after reuse (Figure 7 - Ca1). Hence, several experiments were carried out from which a larger amount of $\mathrm{CaBZ}$ was recovered and recycling experiments were performed under the same conditions, including total reaction volume. Results of $66.49,66.45$ and $66.05 \%$ were obtained in the first, second and third consecutive reaction cycles, respectively, confirming the maintenance of the activity of the catalyst for at least two recycling stages.

The values obtained for the reuse of $\mathrm{CaBZ}$ and SrBZ, under the conditions used in experiments 26 and 47, respectively, revealed the same behavior noted earlier for $\mathrm{BaBZ}$, that is, a slight decrease in the percentage of ester obtained when compared to the conversion of the first reaction cycle using fresh catalyst (Figure 7). Thus, all the compounds showed good conversions after reuse for at least three reaction cycles.

\section{CONCLUSIONS}

The metal benzoates synthesized were found to have layered structures in which four benzoate groups coordinate with different metal sites to form a bidentade bridge. Also, analyses by X-ray powder diffraction showed that these compounds had similar structures. These solid materials were shown to be catalytically active in the esterification of benzoic acid with methanol but, due to their capacity to solubilize in the reaction media, they behaved as homogeneous catalysts that could be recovered and recycled after removal of the excess methanol followed by precipitation with acetone. The catalytic effect of metal benzoates was consistently higher than the corresponding thermal conversion, leading to ester conversions of 65 to $70 \%$ for all the catalysts under the best reaction conditions. The possibility of recycling these metallic benzoates was also demonstrated, evidenced by unaltered catalytic activity for three consecutive reaction cycles. Also, these metal benzoates were able to retain their original layered structure as well as their catalytic properties after use in one or more reaction cycles. These results show that layered metal carboxylates such as calcium, barium and strontium benzoates are stable, environmentally benign and recyclable catalysts that meet the standard of green chemistry for a very important and well established organic reaction. Moreover, their unique properties can be easily adapted to existing industrial facilities based on conventional homogenous catalysis or be used as the active phase for new industrial processes based on heterogeneous catalysts and reactive distillation.

\section{SUPPLEMENTARY MATERIAL}

Available at http://quimicanova.sbq.org.br, in PDF file, with free access. Figure 1S. Thermal analysis curves of the synthesized calcium benzoates - CaBZ; Figure $2 \mathrm{~S}$. Thermal analysis curves of the synthesized strontium benzoates - SrBZ; Figure 3S. X-ray diffraction patterns of calcium benzoate before (a) and after two reaction cycles (b,c); Figure 4S. X-ray diffraction patterns of strontium benzoate before (a) and after two reaction cycles (b,c); Figure 5S. FTIR spectra of calcium benzoate before (a) and after two reaction cycles (b,c); Figure 6S. FTIR spectra of strontium benzoate before (a) and after two reaction cycles $(b, c)$.

\section{ACKNOWLEDGEMENTS}

We are grateful to the Graduate Program in Materials Science and Engineering (PIPE-UFPR), to the funding agencies CNPq, FINEP, CAPES and Araucária Foundation and to LAMIR-UFPR for the TGA/DTA analysis.

\section{REFERENCES}

1. Dudareva, N.; Murfitt, L. M.; Mann, C. J.; Gorenstein, N.; Kolosova, N.; Kish, C. M.; Bonham, C.; Wood, K.; Plant Cell 2000, 12, 949.

2. Farhi, M.; Dudareva, N.; Masci, T.; Weiss, D.; Vainstein, A. H.; J. Biotechnol. 2006, 122, 307.

3. Li, X.; Eli, W.; Li, G.; Catal. Commun. 2008, 2264.

4. Murfitt, L. M.; Kolosova, N.; Arch. Biochem. Biophys. 2000, 1, 145.

5. Parkinson, D.; Churchill, T. J.; Rolls, W.; Bull. Environ. Contam. Toxicol. 2008, 81, 494.

6. Parry, E. J.; The chemistry of essential oils and artificial perfumes, $4^{\text {th }}$ ed., Greenwood and Son: London, 1921.

7. Solomons, T. W. G.; Fryhle, B. C.; Organic Chemistry, $7^{\text {th }}$ ed., John Wiley \& Sons: New York, 2000.

8. Tang, S.; Li, S.; Ind. Eng. Chem. Res. 2004, 43, 6931.

9. Wu, D.; Chang, W.; Wen, X.; Fuel Process. Technol. 2008, 89, 803.

10. Pipus, G.; Plazl, I.; Koloini, T.; Chem. Eng. J. 2000, 76, 239.

11. Cordeiro, C. S.; Arízaga, G. G. C.; Ramos, L. P.; Wypych, W.; Catal. Commun. 2008, 9, 2140. 
12. Musayev, F. N.; Cherkezova, V. R.; Sultanov, B. Y.; Thermochim. Acta 1985, 93, 61.

13. Bruns, R. E.; Scarmínio, I. S.; Barros Neto, B.; Planejamento e otimização de experimentos, $2^{\mathrm{a}}$ ed., Ed. Unicamp: Campinas, 1995.

14. Firestone, D.; Official Methods and Recommended Practices of the AOCS, $6^{\text {th }}$ ed, AOCS: Champaign, 2010.

15. Keli, Z.; Jibing, Y.; Liangjie, Y.; Jutang, S.; Wuhan, J.; Nat. Sci. 1999, 4, 89.

16. Senkovska, I.; Thewalt, U.; Acta Crystallogr. 2005, 61, 448.

17. Terakita, A.; Byrn, S. R.; J. Pharm. Sci. 2006, 95, 1162.

18. Zhang, K.; Yuan, J.; Yuan, L.; Sun, J. J. Therm. Anal. Calorim. 1999, 58, 297.

19. Borawska, M. H.; Koczon, P.; Piekut, J.; Swisłocka, R.; Lewandowski, W.; J. Mol. Struct. 2009, 919, 284.
20. Pavkovic, S. F.; J. Inorg. Nuc. Chem. 1971, 33, 1475.

21. Lisboa, F. S.; Arizaga, G. G. C.; Wypych, F.; Topics Catal. 2011, 54, 474.

22. Spectral database for organic compounds (SDBS-AIST), http://riodb01. ibase.aist.go.jp/sdbs/cgi-bin/cre_index.cgi?lang=eng, accessed on January 2010.

23. Kumar, A.; Easo, S.; Singh, M.; Prasad, B. B.; Polymer 1996, 37, 281

24. Aranda, D. A. G.; Santos, R. T. P.; Tapanes, N. C. O.; Ramos, A. L. D.; Antunes, O. A. C.; Catal. Lett. 2008, 122, 20.

25. Nielsen, R. B.; Kongshaug, K. O.; Fjellvag, H.; J. Mater. Chem. 2008 , 18,1002 . 
ALKALINE EARTH LAYERED BENZOATES AS REUSABLE HETEROGENEOUS CATALYSTS FOR THE METHYL ESTERIFICATION OF BENZOIC ACID

Swamy Arêa Maruyama, Fabio da Silva Lisboa, Luiz Pereira Ramos e Fernando Wypych*

Departamento de Química, Universidade Federal do Paraná, CP 19081, Centro Politécnico, 81531-980 Curitiba, PR - Brasil

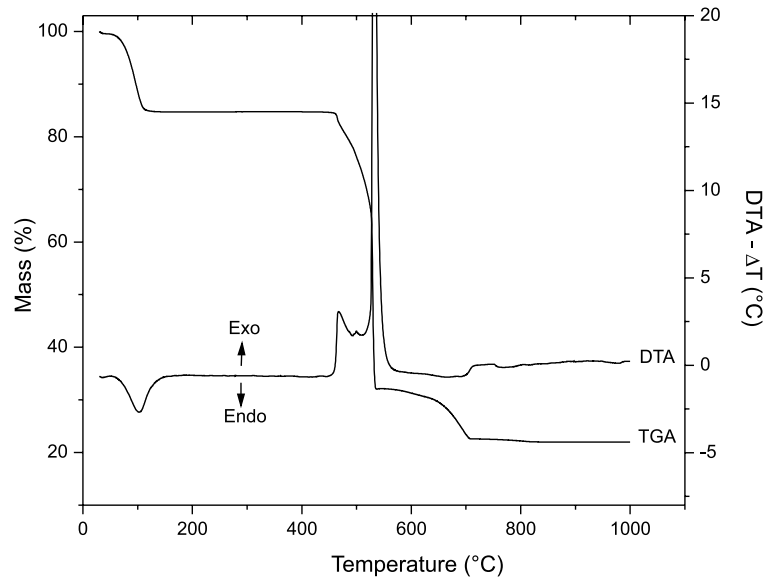

Figure 1S. Thermal analysis curves of the synthesized calcium benzoates $-\mathrm{CaBZ}$

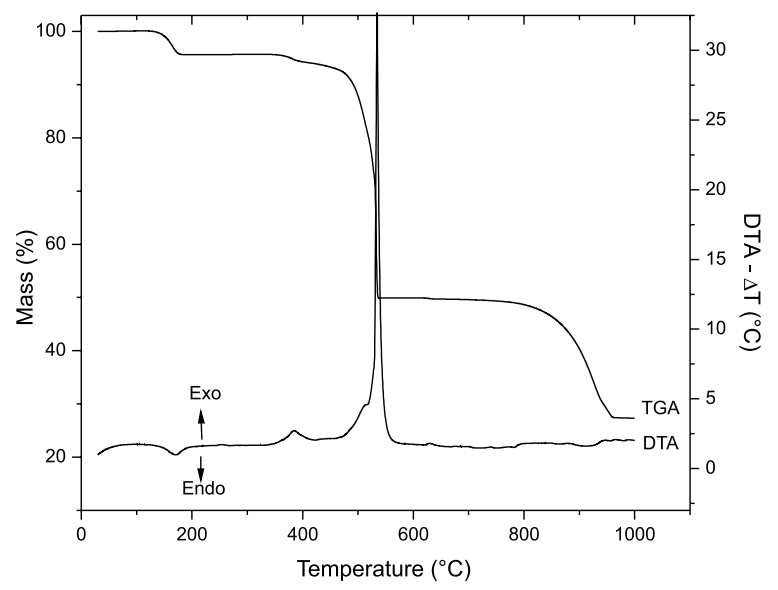

Figure 2S. Thermal analysis curves of the synthesized strontium benzoates $-\operatorname{SrBZ}$

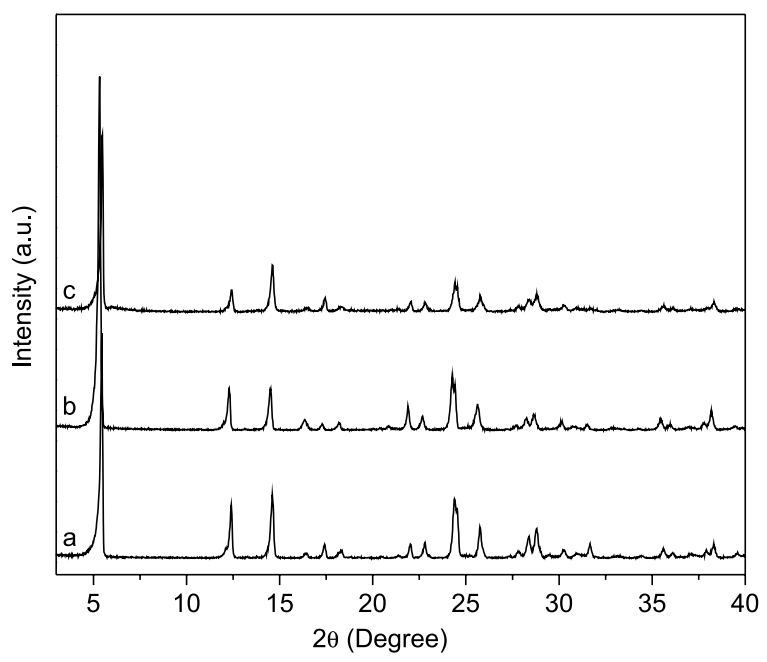

Figure 3S. X-ray diffraction patterns of calcium benzoate before (a) and after two reaction cycles $(b, c)$

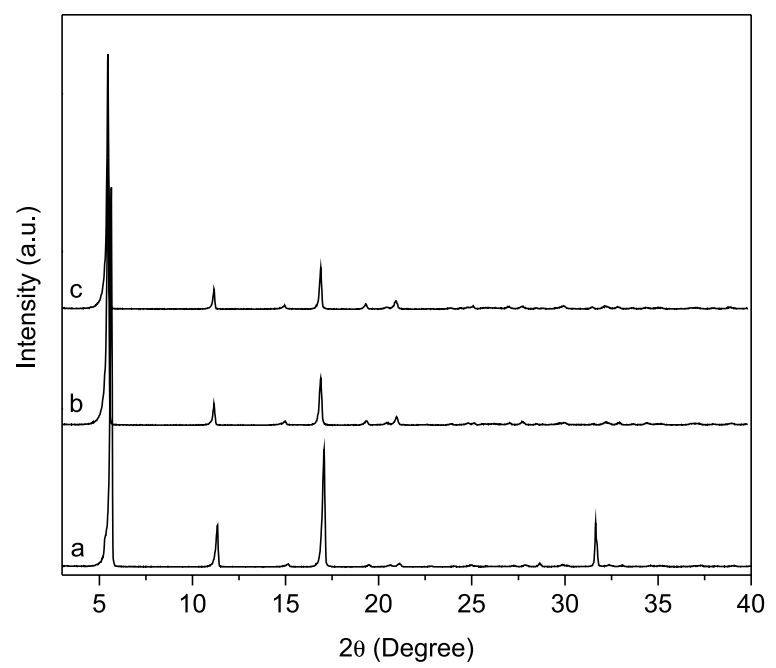

Figure 4S. X-ray diffraction patterns of strontium benzoate before (a) and after two reaction cycles $(b, c)$ 


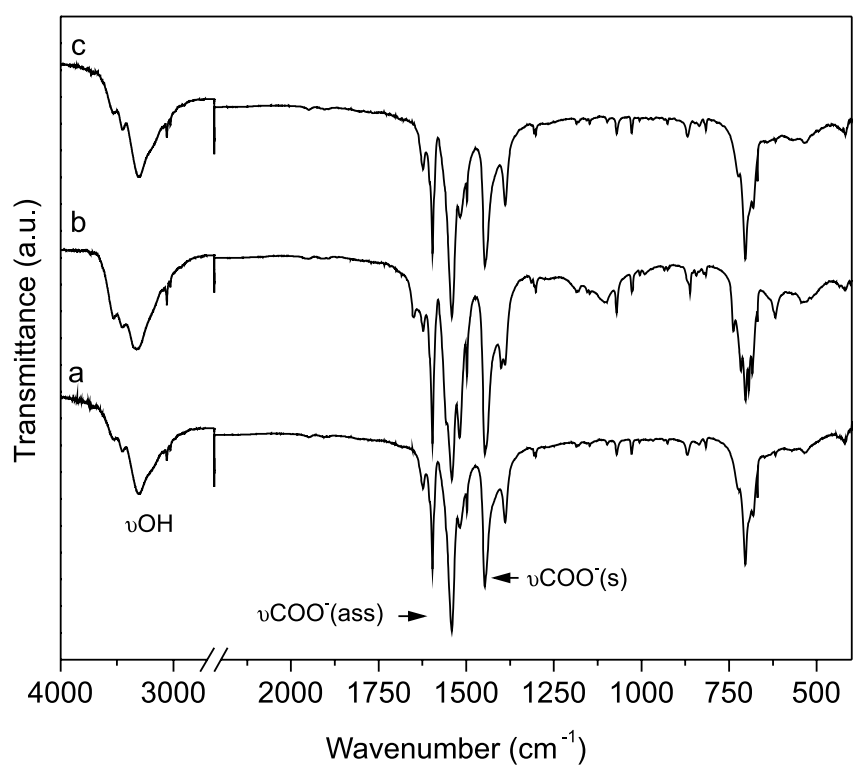

Figure 5S. FTIR spectra of calcium benzoate before (a) and after two reaction cycles $(b, c)$

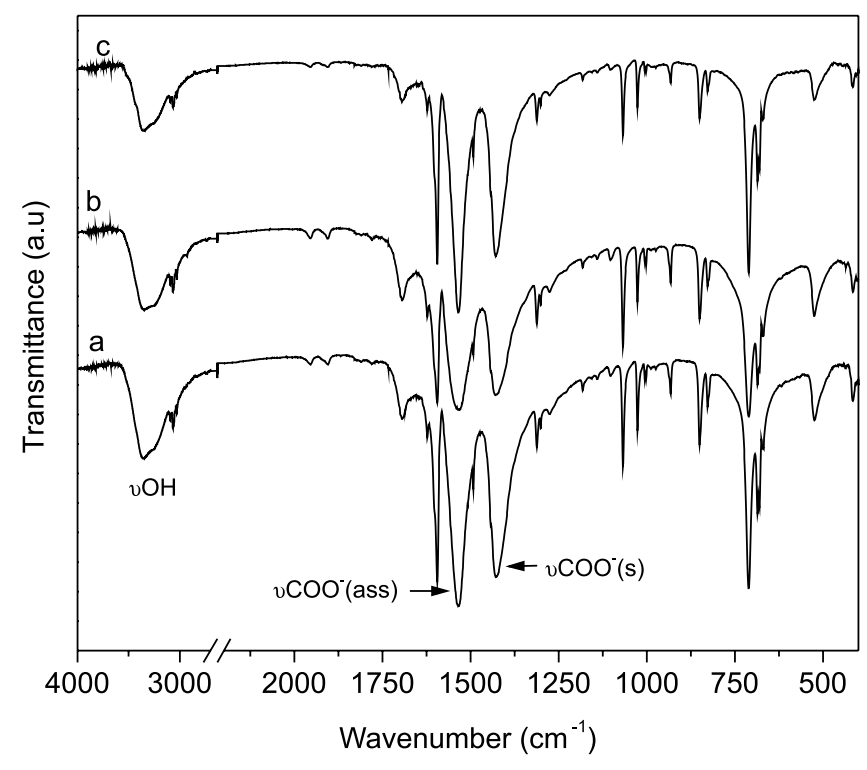

Figure 6S. FTIR spectra of strontium benzoate before (a) and after two reaction cycles $(b, c)$ 